\title{
Lessons learned from the development of an Abl tyrosine kinase inhibitor for chronic myelogenous leukemia
}

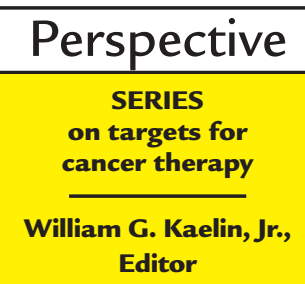

\author{
Brian J. Druker ${ }^{1}$ and Nicholas B. Lydon ${ }^{2}$ \\ ${ }^{1}$ Division of Hematology and Medical Oncology, Oregon Health Sciences University, Portland, Oregon 97201, USA \\ ${ }^{2}$ Kinetix Pharmaceuticals Inc., Medford, Massachusetts 02155, USA \\ Address correspondence to: Nicholas B. Lydon, Kinetix Pharmaceuticals Inc., 200 Boston Avenue, Suite 3500, Medford, \\ Massachusetts 02155, USA. Phone: (781) 391-7577; Fax: (781) 391-5771; E-mail: lydon@kinetixpharm.com.
}

Protein kinases are a large family of homologous proteins comprising 2 major subfamilies, the protein serine/threonine kinases and protein tyrosine kinases (PTKs). Protein kinases function as components of signal transduction pathways, playing a central role in diverse biological processes such as control of cell growth, metabolism, differentiation, and apoptosis. The development of selective protein kinase inhibitors that can block or modulate diseases with abnormalities in these signaling pathways is considered a promising approach for drug development. Because of their deregulation in human cancers, Bcr-Abl, EGFR, HER2, and protein kinase $\mathrm{C}(\mathrm{PKC})$, were among the first protein kinases considered as targets for the development of selective inhibitors. Subsequently, as protein kinases have been implicated in more human cancers (1), drugdiscovery efforts have been extended and several firstgeneration small-molecule inhibitors are now in various stages of development. A selection of these agents is shown in Table 1.

Based on its clear disease association, we saw the BcrAbl tyrosine kinase as an ideal target for validating the clinical utility of protein kinase inhibitors. Here, we dis- cuss our experience in the preclinical and clinical development of a Bcr-Abl inhibitor as a therapeutic agent for chronic myelogenous leukemia (CML), and we consider how this experience and other recent advances in the field could contribute to drug development for other diseases.

\section{The Bcr-Abl kinase as a target}

CML is a hematological stem cell disorder characterized by excessive proliferation of cells of the myeloid lineage. The hallmark of CML is the Philadelphia chromosome, which arises from a reciprocal translocation between chromosomes 9 and 22 (2). The molecular consequence of this translocation is the replacement of the first exon of $c-A b l$ with sequences from the $B c r$ gene resulting in a $B c r-A b l$ fusion gene whose protein product shows enhanced tyrosine kinase activity (3-7) (Figure 1). The Bcr-Abl oncoprotein in CML is a $210-\mathrm{kD}$ protein that contains 902 or 927 amino acids of Bcr fused to exons $2-11$ of c- $A b l(5,6)$. Found in $95 \%$ of patients with CML, p210Bcr-Abl is also present in approximately $5-10 \%$ of adults with acute leukemia for whom there is no evidence of antecedent CML (8). Another Bcr-Abl fusion protein of $185 \mathrm{kD}$ containing $\mathrm{Bcr}$ sequences from exon 1

Table 1

Selected small-molecule ATP-competitive protein kinase inhibitors in development

\begin{tabular}{|c|c|c|c|}
\hline Target kinase $^{\mathrm{A}}$ & Company & Name & Clinical phase \\
\hline Bcr-Abl & Novartis & CGP 57148B (STI 571) & Phase I/II \\
\hline CDKs & Hoechst Marion Roussel & (L86-8275, NSC-649890) & Phase I \\
\hline EGF-R & Boehringer-Ingelheim & BIBX 1382 & Phase I \\
\hline EGF-R & Novartis & PKI166 & Phase I \\
\hline EGF-R & Pfizer/OSI Pharmaceuticals & CP 358,774 & Phase II \\
\hline EGF-R & Warner-Lambert & PD 0183805 & Phase I \\
\hline EGF-R & Zeneca & ZD 1839 & Phase II \\
\hline $\mathrm{PKC} /$ Trk $^{\mathrm{B}}$ & $\begin{array}{c}\text { Cephalon/ } \\
\text { Kyowa Hakko }\end{array}$ & $\begin{array}{l}\text { CEP } 2563 \\
\text { CEP } 701\end{array}$ & Phase I \\
\hline PKC ${ }^{\mathrm{B}}$ & Kyowa Hakko & UCN-01 & Phase I/II \\
\hline PKC $\beta$ & Lilly & LY-333531 & Phase III \\
\hline $\mathrm{PKC} C^{\mathrm{B}}$ & Novartis & CGP 41251 (STI 412) & Phase I/II \\
\hline PDGF-R $\beta$ & Sugen & SU 6668 & Phase I \\
\hline VEGF-R & Novartis/Schering & $\begin{array}{c}\text { CGP 79787 } \\
\text { (PTK787/ZK22584) }\end{array}$ & Phase I \\
\hline VEGF-R & Pfizer/OSI Pharmaceuticals & CP-564 959 & PCD \\
\hline VEGF-R & Sugen & SU 5416 & Phase II \\
\hline VEGF-R & Zeneca & ZD 4190 & PCD \\
\hline
\end{tabular}

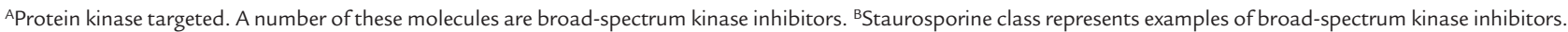
PCD, preclinical development. These compounds may have progressed to phase I clinical trials. 


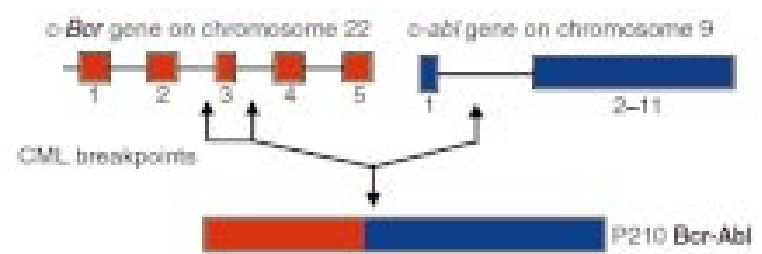

Figure 1

Structure of the $B c r-A b /$ gene. The Philadelphia chromosome is formed by a reciprocal translocation between chromosomes 9 and 22. Potential breakpoints are indicated by arrows. This resulting translocation replaces the first exon of c-ab/ with sequences from the Bcr gene.

(426 amino acids) fused to exons $2-11$ of c- $A b l$, occurs in $10 \%$ of adult cases and $5-10 \%$ of pediatric cases of acute lymphoblastic leukemia, but not in $\operatorname{CML}(9,10)$.

The $A b l$ oncogene was isolated originally from the genome of the Abelson murine leukemia virus (A-MuLV) (11). This acutely transforming replication-defective virus encodes a transforming protein ( $160 \mathrm{v}-\mathrm{Abl})$ with tyrosine-specific protein kinase activity. A-MuLV transforms fibroblasts in vitro and lymphoid cells in vitro and in vivo and was formed by recombination between Moloney murine leukemia virus (M-MuLV) and the murine c- $A b l$ gene (11).

Expression of p210Bcr-Abl induces a disease resembling CML in mice $(12,13)$, confirming that the Bcr-Abl oncoprotein is a major factor in the pathophysiology of CML. Additional studies have shown that PTK activity is essential to the transforming function of $\mathrm{Bcr}-\mathrm{Abl}$ (14). Thus, the presence of Bcr-Abl in the majority of CML patients, and the requirement of kinase activity for BcrAbl function, make this a particularly attractive target for design of a selective kinase inhibitor.

\section{Pharmacological profile of STI 571}

Having identified an appropriate target, the next task was to design an inhibitor of this enzyme. The 2-phenylaminopyrimidines were first reported as potent PTK inhibitors with selectivity for the Abl and PDGF-R tyrosine kinases $(15,16)$. As is the case with many of the inhibitors currently in clinical trials, an initial lead compound was identified by the time-consuming process of random screening, that is, the testing of large compound libraries for inhibition of protein kinases in vitro. In this case, the initial lead compound was a relatively weak inhibitor of PKC $\alpha$ and the PDGF-R (17). The activity of the 2-phenylaminopyrimidine series was optimized for inhibition of the PDGF-R by synthesizing a series of chemically related compounds and analyzing the relationship between their structure and activity. The most potent molecules in the series were all dual inhibitors of the $\mathrm{v}$-Abl and the PDGF-R kinases. STI 571 (formerly CGP 57148B) emerged from these efforts as the lead compound for preclinical development.

STI 571 has been tested in a number of preclinical models. We found that submicromolar concentrations of the compound inhibited autophosphorylation of $\mathrm{v}$-Abl, PDGF receptor, and Kit receptor and blocked PDGF-induced inositol phosphate formation, MAP kinase activation, and c-fos mRNA expression in intact cells $(15,16)$.
In a pivotal set of preclinical experiments, STI 571 was shown to suppress the proliferation of Bcr-Abl-expressing cells in vitro and in vivo (18). In colony-forming assays of peripheral blood or bone marrow from patients with CML, STI 571 caused a 92-98\% decrease in the number of Bcr-Abl colonies formed, with minimal inhibition of normal colony formation (18). Our cellular in vivo and human ex-vivo studies convinced us that STI 571 could be useful in diseases involving deregulated Abl PTK activity.

The efficacy and specificity of STI 571 has been confirmed and extended by several laboratories (19-21). We and others have also demonstrated activity of STI 571 against $\mathrm{p} 185 \mathrm{Bcr}-\mathrm{Abl}$ and another activated $\mathrm{Abl}$ fusion protein, Tel-Abl $(19,22)$.

From laboratory to clinical concept validation in CML We initially supposed that continual suppression of the Bcr-Abl tyrosine kinase would be required for maximal clinical benefit in CML. Indeed, in our early studies we found that Bcr-Abl-expressing cells could be rescued from apoptotic cell death if STI 571 was washed out of the cells within 16 hours of initial exposure. If a tyrosine kinase inhibitor inhibited proliferation of Bcr-Abl-positive cells without inducing cell death, then long-term therapy might be required, suggesting that a well-tolerated, oral formulation of the drug would be needed.

Early pharmacokinetic studies in rats and dogs demonstrated that bioactive concentrations of compound are readily achieved in the circulation. Following up on these findings, le Coutre et al. treated Bcr-Abl tumor-bearing nude mice with a regimen that assured a continuous block of Bcr-Abl kinase activity (21). Administration of STI 5713 times per day, over an 11-day period, cured $87-100 \%$ of treated mice, whereas administration once or twice per day did not. This confirmed our hypothesis that continuous exposure may be critical to the success of this inhibitor as a therapeutic agent.

Toxicity testing by daily oral administration to rats for 13 weeks showed occasional renal calcifications and mild bladder mucosal hyperplasia at the lowest dose of $6 \mathrm{mg} / \mathrm{kg}$. In dogs, a no-effect level was seen at $3 \mathrm{mg} / \mathrm{kg}$, but progressive liver toxicity was seen at the highest dose of $100 \mathrm{mg} / \mathrm{kg}$. At the highest dose levels, some vomiting, diarrhea, mild anemia, and neutropenia were also observed.

Based on the preclinical data, we designed a phase I/II study to test the tolerability and pharmacokinetic properties of the compound and to look for preliminary signs of efficacy. These studies have been ongoing since June 1998 and have targeted chronic-phase CML patients who are resistant to interferon therapy. Treatment with STI 571 is given as daily oral therapy. Side effects have been minimal, and no dose-limiting toxicities have been encountered. At doses of $300 \mathrm{mg}$ or greater, all patients have achieved complete hematologic responses, and cytogenetic responses have also been observed (23). Pharmacokinetics demonstrate that STI 571 has a half-life of between 12 and 14 hours, and the concentrations predicted to be effective in preclinical studies correlate well with the clinical responses.

\section{Future prospective and issues}

Our experience with the Abl kinase inhibitor dramatically demonstrates the potential of molecularly target- 
ed therapy. However, CML and Bcr-Abl have several unique advantages that will be discussed. Other areas of investigation that deserve specific comment include identification and validation of targets and surrogate endpoints, improved efficiency of drug design, and desirability of inhibitor specificity.

\section{Target identification and therapeutic concept}

Given the ability to design specific kinase inhibitors, one of the major issues is to identify appropriate targets for drug development. Although the Abl kinase inhibitor has been useful for clinical concept validation, several features of CML may make the success of a kinase inhibitor as a single agent unique for this cancer. The Bcr-Abl tyrosine kinase, present in $95 \%$ of patients, is sufficient to cause the disease, and in early disease, it may represent the sole molecular abnormality. Few other malignant diseases can be ascribed to a single molecular defect in a protein kinase. Other examples may include Alk in anaplastic large-cell lymphoma, Ret in multiple endocrine neoplasia, Tel- PDGF-R in chronic myelomonocytic leukemia and possibly others (1). However, the emerging field of disease genomics will likely identify other members of the protein kinase family as candidate disease genes. Once candidate kinase genes are identified, it will be critical to show that a defect in the kinase can cause the specific disease in animal or other model systems.

In addition, many nonmalignant, proliferative, or autoimmune diseases may be caused by single activated protein kinases and would therefore prove to be suitable targets for protein kinase inhibitors. Kinases whose function appears to be redundant or expressed in a tissuespecific manner would be particularly attractive targets, whereas other kinases that are critical for normal cellular function may be more difficult to target safely. Kinases in the former group include those associated with antigen signaling in T cells, such as ZAP70, Lck, Itk, Fyn.

Although specific kinase inhibitors are likely to be of maximal therapeutic benefit when their target kinase is the sole causative abnormality, such compounds may be useful in other, more complicated disorders. In these instances kinase inhibitors may need to be combined with standard therapies or, perhaps, other molecularly targeted therapeutics.
Identification and validation of surrogate endpoints Another attractive feature of CML for clinical concept validation was that the disease can be easily monitored through analysis of blood counts. In the majority of patients being treated in the study, the white blood count has been restored to the normal range within 3 weeks of initiation of therapy and has remained normal with continued therapy. The presence of the Philadelphia chromosome can be used as a surrogate of disease activity. In this regard, the cytogenetic responses suggest that Bcr-Abl-expressing cells either have a selective growth disadvantage or they undergo apoptotic cell death in the presence of STI 571.

In our clinical trial, the concentration of drug required to kill leukemia cells ex-vivo could be used directly as a target dose in phase I studies simply because CML is a disease of the blood. In solid tumors, blood levels may or may not be predictive of tumor drug levels, so it will be essential to develop other markers of clinical endpoints to determine optimal dosing of these novel agents. This is particularly important, given the potential difficulty in defining maximally tolerated doses for such agents.

With STI 571, assessing for inhibition of the Bcr-Abl tyrosine kinase has been somewhat problematic, and we are currently developing phospho-Abl-specific reagents for this purpose. In the meantime, we have had some success analyzing inhibition of tyrosine phosphorylation of substrates of Bcr-Abl such as Crkl, which is the major novel tyrosine phosphorylated protein in CML-patient neutrophils (24). We believe that the difficulty of using kinase inhibition as a surrogate marker will present a major, but not insurmountable, challenge when attempting to monitor more complex and heterogeneous malignancies, especially solid tumors.

\section{Improved efficiency of drug design and specificity of inhibition}

Because all members of the kinase family bind the same nucleotide cofactor, ATP, the dogma in the field had been that the ATP-binding cleft (Figure 2) of this enzyme class would not prove to be a good target for drug discovery (25). A number of developments have put this concern to rest. The most persuasive data include the

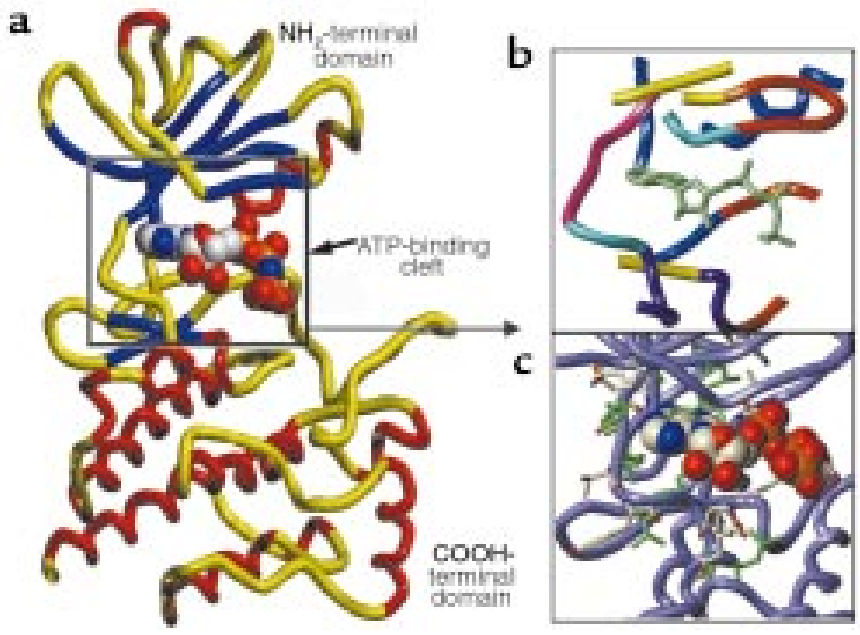

\begin{abstract}
Figure 2
The crystal structure of AMP-PNP bound to Lck was used to made a model of the Abl kinase domain. (a) The general architecture of the Abl kinase domain. The $\mathrm{NH}_{2}$-terminal domain has a 5 -strand $\beta$-sheet (blue) and $1 \alpha$-helix (red). The $\mathrm{COOH}$-terminal domain is made up of several $\alpha$-helices. ATP is shown in the ATPbinding cleft. (b) Topology of the ATP-binding cleft of the Abl protein kinase. The ATP-binding cleft can be subdivided into 6 regions: adenine-binding region (yellow), hydrophobic region I (blue), hydrophobic region II (aqua), phosphate-binding region (red-orange), the ribose pocket (purple), and the linker region (pink). The ligated ATP is shown in green-blue. (c) Amino acid side chains lining the ATP-binding cleft.
\end{abstract}


a

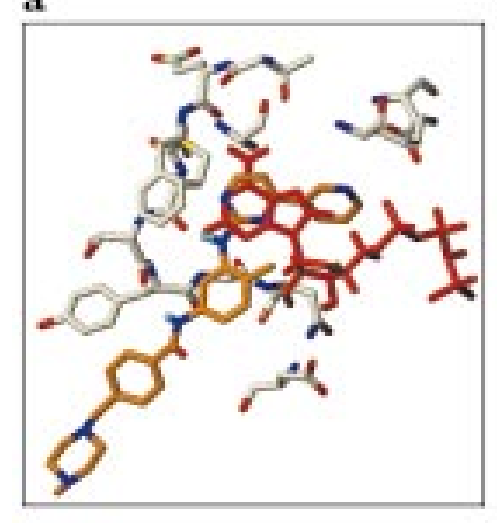

b

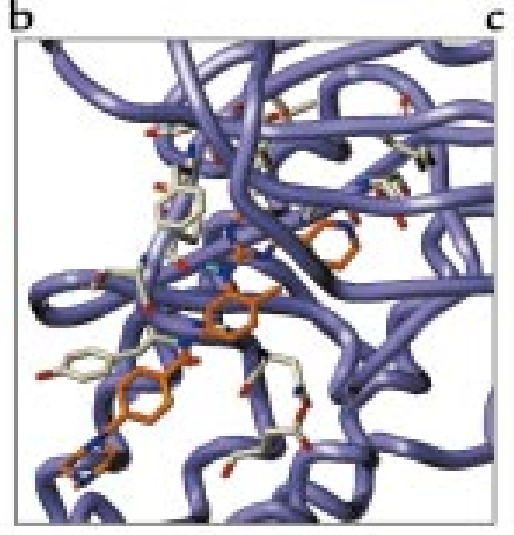

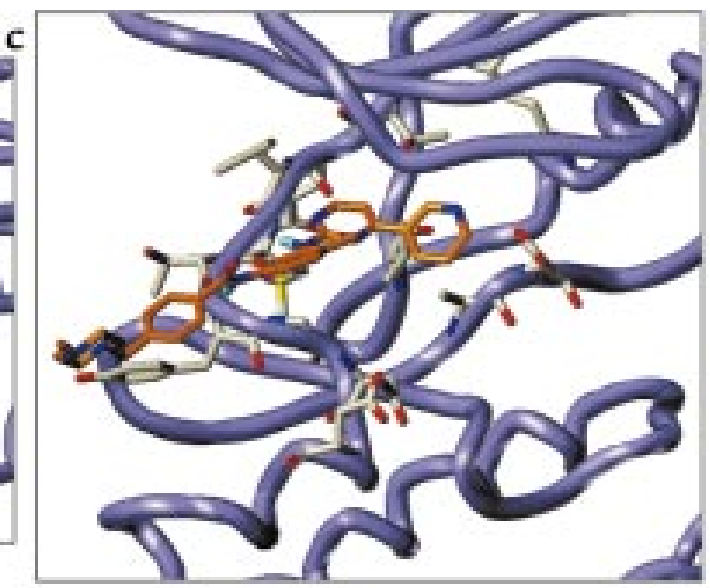

Figure 3

Model of STI 571 bound within the active site of the Abl kinase. (a) Overlay of ATP (red) and STI 571 (orange). Key amino acids that interact with ATP are shown. Binding mode of STI 571 shown from above (b) and from the side (c).

discovery and clinical development of highly selective EGFR inhibitors of the quinazoline, pyrrolopyrimidine and pyrazolopyrimidine classes (26).

The second development has been the elucidation of inhibitor mechanisms and specificity, based on crystallographic data (25). Recent studies reporting the structures of kinases in complex with selective ATP site-directed inhibitors reveal that these selective inhibitors make contacts with both conserved and nonconserved residues within the ATP-binding site. Nonconserved residues are located in pockets that do not bind ATP and are likely to be unique to subsets of protein kinases. Together, these results provide increased confidence that developing inhibitors directed at the ATP-binding cleft is a viable approach to selectively inhibiting kinases.

The number of $x$-ray crystal structures of protein kinases has increased dramatically. There are presently over 60 protein kinase structures in the Protein Data Bank. These structures include both apo-enzymes and binary complexes with ATP, the ATP-analog AMP-PNP, or small-molecule inhibitors, either with or without peptide substrate. Several of these crystal structures are complexes of kinases with relatively selective kinase inhibitors. The availability of this structural information from multiple members of the protein kinase family allows one to see how small changes in the primary structure, even those remote from the ATP-binding site, can be exploited to develop selective kinase inhibitors. An example of this has been provided by the crystallization of closely related pyrimidine analogs PP1 and PP2 bound to both lck (27) and Hck (28). The accumulating structural and chemical data is expected to dramatically accelerate the discovery and optimization of selective protein kinase-inhibitor drugs against a variety of new kinase targets. Additionally, with structural information in hand, physicochemical features, such as solubility, can be optimized using positions on the inhibitor molecule that do not compromise potency or selectivity.

In the absence of structural information, no clear explanation for the impressive selectivity of STI 571 could be made. Retrospective analysis by modeling stud- ies now enables us to propose a binding mode for STI 571 that can explain much of the specificity of the compound. A model of the Abl kinase domain based on the $\mathrm{x}$-ray structure of Lck (27) is shown in Figure 2. The proposed binding mode of STI 571 is shown in Figure 3 from 2 angles (Figure 3, a and b). A comparison of the binding of ATP and STI 571 is shown in Figure 2c. The specificity of the molecule can be explained by the combination of interactions that STI 571 makes with the active site of the Abl enzyme.

Depending on the intended clinical use and therapeutic window required, different selectivity profiles may be needed for optimal clinical activity of a kinase inhibitor. In nonmalignant diseases a higher degree of selectivity is likely to be required to achieve an acceptable therapeutic window. Similarly, the side-effect profile that results from inhibiting various kinases will need to be considered in deciding whether to optimize specificity. For example, if a broadly expressed kinase that has a critical activity for normal cellular function is part of the spectrum of inhibition of a kinase, but this kinase is not the intended target, it would seem desirable to further optimize an inhibitor such that this unintended activity is removed.

In other cases, it may be beneficial to design molecules that have a broad spectrum of activity. In breast cancer, both Her-2 and the EGF receptor have been reported to contribute to the malignant phenotype. Thus, combining inhibitors of each of these kinases or developing an inhibitor with dual specificity may be more effective than a specific inhibitor of only one of these kinases. Similarly, in the case of STI 571, further profiling has shown that it also inhibits c-kit, the receptor for stem cell factor, a cytokine involved in hematopoiesis. It is plausible that the combination of $\mathrm{Abl}$ and c-kit inhibition may contribute to the in vivo activity of the compound in CML.

\section{Conclusions}

The challenge for molecular biologists is how to harness the wealth of structural data in the field so that series (or focused libraries) of molecules can be identified and derivatized for selectivity against the intended targets. 
Because of the large number of potential targets, which will increase as genome-based methods identify more candidates, this endeavor requires the integration of a number of disciplines, including structural biology, computational chemistry, structurally directed medicinal chemistry, array screening assays, and molecular and cellular biology. Additional efforts will also be required for the analysis and validation of surrogate molecular endpoints. Through these efforts, we believe that the $\mathrm{Bcr}-\mathrm{Abl}$ kinase inhibitor story will be but the first example of many future successes.

\section{Acknowledgments}

We gratefully acknowledge Leticia Toledo for Figures 2 and 3; Jürg Zimmermann, Elizabeth Buchdunger, and the STI 571 project team; and Kathryn Kolibaba for critcally reading the manuscript.

1. Kolibaba, K.S., and Druker, B.J. 1997. Protein tyrosine kinases and cancer. Biochim. Biophys. Acta. 1333:F217-F248.

2. Rowley, J.D. 1973. A new consistent abnormality in chronic myelogenous leukaemia identified by quinacrine fluorescence and giemsa staining. Nature. 243:290-293.

3. Heisterkamp, N., et al. 1983. Localization of the c-abl oncogene adjacent to a translocation break point in chronic myelocytic leukemia. Nature. 306:239-242.

4. Bartram, C.R., et al. 1983. Translocation of c-abl correlates with the presence of a Philadelphia chromosome in chronic myelocytic leukemia. Nature. 306:277-280.

5. Shtivelman, E., Lifshitz, B., Gale, R.P., and Canaani, E. 1985. Fused transcript of abl and bcr genes in chronic myelogenous leukaemia. Nature. 315:550-554.

6. Ben-Neriah, Y., Daley, G.Q., Mes-Masson, A.-M., Witte, O.N., and Baltimore, D. 1986. The chronic myelogenous leukemia-specific P210 protein is the product of the bcr/abl hybrid gene. Science. 233:212-214.

7. Konopka, J.B., Watanabe, S.M., and Witte, O.N. 1984. An alteration of the human c-abl protein in K562 unmasks associated tyrosine kinase activity. Cell. 37:1035-1042.

8. Kurzrock, R., Gutterman, J.U., and Talpaz, M. 1988. The molecular genetics of Philadelphia chromosome-positive leukemias. N. Engl.J. Med. 319:990-998.

9. Hermans, A., et al. 1987. Unique fusion of bcr and c-abl genes in Philadelphia chromosome positive acute lymphoblastic leukemia. Cell. 51:33-40.

10. Clark, S.S., et al. 1988. Expression of a distinctive BCR-ABL oncogene in Ph1-positive acute lymphocytic leukemia (ALL). Science. 239:775-777.

11. Rosenberg, N., and Witte, O.N. 1988. The viral and cellular forms of the
Abelson (abl) oncogene. Adv. Virus Res. 35:39-81.

12. Daley, G.Q., Van Etten, R.A., and Baltimore, D. 1990. Induction of chronic myelogenous leukemia in mice by the P210bcr/abl gene of the Philadelphia chromosome. Science. 247:824-830.

13. Kelliher, M.A., McLaughlin, J., Witte, O.N., and Rosenberg, N. 1990. Induction of a chronic myelogenous leukemia-like syndrome in mice with v-abl and BCR/ABL. Proc. Natl. Acad. Sci. USA. 87:6649-6653.

14. Lugo, T.G., Pendergast, A.M., Muller, A.J., and Witte, O.N. 1990. Tyrosine kinase activity and transformation potency of bcr-abl oncogene products. Science. 247:1079-1082.

15. Buchdunger, E., et al. 1995. Selective inhibition of the platelet-derived growth factor signal transduction pathway by a protein-tyrosine kinase inhibitor of the 2-phenylaminopyrimidine class. Proc. Natl. Acad. Sci. USA 92:2558-2562.

16. Buchdunger, E., et al. 1996. Inhibition of the Abl protein-tyrosine kinase in vitro and in vivo by a 2-phenylaminopyrimidine derivative. Cancer Res. 56:100-104.

17. Zimmermann, J., et al. 1996. Phenylamino-pyrimidine (PAP) derivatives: a new class of potent and selective inhibitors of protein kinase C (PKC). Arch. Pharm. (Weinheim). 329:371-376.

18. Druker, B.J., et al. 1996. Effects of a selective inhibitor of the ABL tyrosine kinase on the growth of BCR-ABL positive cells. Nat. Med. 2:561-566.

19. Carroll, M., et al. 1997. CGP 57148, a tyrosine kinase inhibitor, inhibits the growth of cells expressing BCR-ABL, TEL-ABL and TEL-PDGFR fusion proteins. Blood. 90:4947-4952.

20. Deininger, M.W., Goldman, J.M., Lydon, N., and Melo, J.V. 1997. The tyrosine kinase inhibitor CGP57148B selectively inhibits the growth of BCR-ABL-positive cells. Blood. 90:3691-3698.

21. le Coutre, P., et al. 1999. In vivo eradication of human BCR/ABL-positive leukemia cells with an ABL kinase inhibitor. J. Natl. Cancer Inst. 91:163-168.

22. Beran, M., et al. 1998. Selective inhibition of cell proliferation and BCR$\mathrm{ABL}$ phosphorylation in acute lymphoblastic leukemia cells expressing Mr 190,000 BCR-ABL protein by a tyrosine kinase inhibitor (CGP57148). Clin. Cancer Res. 4:1661-1672.

23. Druker, B.J., et al. 1999. Clinical efficacy and safety of an Abl specific tyrosine kinase inhibitor as targeted therapy for chronic myelogenous leukemia. Blood. 94:368a.

24. Oda, T., et al. 1994. CRKL is the major tyrosine phosphorylated protein in neutrophils from patients with chronic myelogenous leukemia. J. Biol. Chem. 269:22925-22928.

25. Toledo, L.M., Lydon, N.B., and Elbaum, D. 1999. The structure-based design of ATP-site directed protein kinase inhibitors. Curr. Med. Chem. 6:775-805.

26. Traxler, P., et al. 1997. Use of a pharmacophore model for the design of EGF-R tyrosine kinase inhibitors: 4-(phenylamino)pyrazolo[3,4d] pyrimidines. J. Med. Chem. 40:3601-3616.

27. Zhu, X., et al. 1999. Structural analysis of the lymphocyte-specific kinase Lck in complex with non-selective and Src family selective kinase inhibitors. Structure Fold Des. 7:651-661.

28. Schindler, T., et al. 1999. Crystal structure of Hck in complex with a Src family-selective tyrosine kinase inhibitor. Mol. Cell. 3:639-648. 